\title{
Cannabinoids and Schizophrenia: Risks and Therapeutic Potential
}

\author{
Marc W. Manseau ${ }^{1}$ - Donald C. Goff ${ }^{1}$
}

Published online: 27 August 2015

(C) The American Society for Experimental NeuroTherapeutics, Inc. 2015

\begin{abstract}
A convergence of evidence shows that use of Cannabis sativa is associated with increased risk of developing psychotic disorders, including schizophrenia, and earlier age at which psychotic symptoms first manifest. Cannabis exposure during adolescence is most strongly associated with the onset of psychosis amongst those who are particularly vulnerable, such as those who have been exposed to child abuse and those with family histories of schizophrenia. Schizophrenia that develops after cannabis use may have a unique clinical phenotype, and several genetic polymorphisms may modulate the relationship between cannabis use and psychosis. The endocannabinoid system has been implicated in psychosis both related and unrelated to cannabis exposure, and studying this system holds potential to increase understanding of the pathophysiology of schizophrenia. Anandamide signaling in the central nervous system may be particularly important. $\Delta^{9}$ Tetrahydrocannabinol in cannabis can cause symptoms of schizophrenia when acutely administered, and cannabidiol (CBD), another compound in cannabis, can counter many of these effects. CBD may have therapeutic potential for the treatment of psychosis following cannabis use, as well as schizophrenia, possibly with better tolerability than current antipsychotic treatments. CBD may also have antiinflammatory and neuroprotective properties. Establishing
\end{abstract}

Electronic supplementary material The online version of this article (doi:10.1007/s13311-015-0382-6) contains supplementary material, which is available to authorized users.

Donald C. Goff

Donald.Goff@nyumc.org

1 Department of Psychiatry, New York University Langone Medical Center, New York, NY, USA the role of CBD and other CBD-based compounds in treating psychotic disorders will require further human research.

Key Words Cannabinoids · schizophrenia $\cdot$ psychosis · cannabidiol $\cdot$ cannabis $\cdot$ endocannabinoid

\section{Introduction}

Schizophrenia is a chronic and often disabling developmental brain disease that affects approximately $1 \%$ of the population over the course of life [1], and is characterized by positive symptoms (delusions, hallucinations, and disorganized thinking), negative symptoms (social withdrawal, amotivation, and affective flattening), deficits in cognition (memory, executive functioning, and processing speed), and a decline in social and occupational functioning [2]. While significant advances have been made in understanding the neurobiology of schizophrenia, and effective treatments have been developed for positive symptoms, the goal of reversing disability associated with cognitive deficits and amotivation has not been realized.

Cannabis sativa is the most commonly used illicit substance globally, and its main psychoactive component, $\Delta^{9}$ tetrahydrocannabinol (THC), has been widely recognized for its ability to cause acute psychotic symptoms and cognitive impairment similar to schizophrenia [3, 4]. Cannabis is also the most commonly abused illicit substance amongst people with schizophrenia, and there is substantial evidence that continued use after a schizophrenia diagnosis is associated with worsening psychotic symptoms, relapse, and decreased functioning over time $[4,5]$. Furthermore, epidemiologic evidence has accumulated implicating cannabis use as an important environmental risk factor for developing schizophrenia. In 1987, Andreasson et al. [6] reported that heavy cannabis use was associated with a 6-fold increase in risk for schizophrenia, 
based on a 15-year follow-up of Swedish military conscripts. Debate has since ensued over whether this association is causal. A greater understanding of the endocannabinoid system (ECS) and its links to psychosis has the potential to generate new insights into the neurobiological causes of schizophrenia, and to open up novel and more efficacious treatment options for this debilitating illness.

Here, we first review the evidence for cannabis use as a causal factor for the development of schizophrenia. Next, we review the biology of the ECS as it pertains to psychosis. Finally, we review preliminary evidence for the use of cannabinoid-based compounds in the treatment of psychosis, and identify potential future research directions for the field.

\section{Cannabis and Schizophrenia}

Evidence for a relationship between cannabis use and risk for psychosis has been replicated frequently. An analysis of results from 35 studies revealed a pooled odds ratio of 1.4 for risk of developing psychosis in individuals with any history of cannabis exposure and an odds ratio of 2.1 among individuals with histories of more frequent use [7]. In addition, a relationship between the age of initiation of cannabis use and age of onset of psychosis has also been well documented, with most studies finding that cannabis use precedes onset of psychosis and that the amount of cannabis exposure in adolescence may be the strongest determinant of this association [4]. However, several issues have complicated interpretation of these findings, including the role of comorbid substance use, of male sex (which is associated with higher rates of cannabis use and earlier age of onset of schizophrenia), of lower socioeconomic status, and of the potentially poor reliability of self-reported histories of past cannabis use. In addition, rather than a causal association, it is possible that cannabis use may represent selfmedication of early symptoms of a psychotic diathesis (e.g., anxiety) or that both the predilection for early use of cannabis and the risk for psychosis are related to a third factor [8]. For example, the high rates of cigarette smoking in individuals with schizophrenia represents a strong association, which is not viewed as causal, but rather is interpreted as reflecting a third factor, dysregulation of nicotinic acetycholine transmission in schizophrenia [9]. Consistent with this model, schizophrenia risk alleles have been linked both to risk for cannabis use and the quantity of use among the general population [10].

In a meta-analysis that included 83 studies and 22,000 individuals with psychotic disorders, substance use was significantly associated with an earlier onset of psychosis (by a mean of 2.7 years), after controlling for sex [11]. Alcohol use did not predict age of onset, whereas the relationship of other substances of abuse to age of onset could not be determined, nor were the authors of the meta-analysis able to restrict subjects to those for whom cannabis use was known to have preceded onset of illness. Interestingly, the relationship between cannabis use and age of onset did not differ between schizophrenia and affective psychosis, suggesting a lack of specificity for schizophrenia. In a study of 625 patients with a first episode of psychosis, subjects whose substance use was restricted to cannabis had an earlier age of onset of psychosis, but only if cannabis use started before the age of 14 years [12]. Whereas the effect of cannabis use before the age of 14 years on age of psychosis onset was small, in the entire sample the age of onset of cannabis strongly predicted age of onset of psychosis, accounting for $25 \%$ of the variance of age of illness onset. Similarly, in a sample of 997 subjects, Stefanis et al. [13] found that onset of cannabis use preceded onset of psychosis on average by 7-8 years regardless of the age at which cannabis use began. This finding is not consistent with the widely held view that adolescence is a unique developmental period of vulnerability to cannabis, but rather that early exposure to cannabis predicts early onset of illness in part because the temporal onset of the 2 are related, regardless of the age at which cannabis use starts. Two longitudinal cohort studies utilized repeated assessments during adolescence and young adulthood to examine the temporal relationship between cannabis use and psychosis [14, 15]. Although the numbers of individuals with psychosis were small, both studies found a bidirectional relationship, such that early psychotic symptoms predicted later cannabis use and vice versa, supporting both a causal relationship and a self-medication hypothesis, or the existence of a third factor. In contrast, Henquet et al. [16] prospectively followed 2437 adolescents and young adults for 4 years and found that low levels of psychotic symptoms in combination with cannabis use predicted onset of psychosis; the risk of psychosis correlated with the frequency of cannabis use. However, low levels of psychotic symptoms did not predict subsequent initiation of cannabis use in participants who were abstinent at baseline. The issue of confounding socioenvironmental factors was addressed by McGrath et al. [17], who used sibling pairs in a study that corroborated the relationship between duration of cannabis use and risk of psychosis [17]. Duration since first cannabis use and subsequent psychosis-related outcome measures remained significant within sibling pairs, suggesting that this relationship is less likely to be explained entirely by unmeasured confounding.

The fact that only a very small minority of cannabis users develop schizophrenia, as well as the absence of an increase in the incidence of psychosis commensurate with the recent population-level increase in cannabis use, suggest that the epidemiologic association between the 2 might be based on confounding factors and/or reverse causation $[18,19]$. Arguing in support of a causal relationship is the consistently replicated evidence in different populations that cannabis use precedes psychotic symptoms [20,21], there is a dose effect [16], and there are coherent, plausible biological mechanisms [4, 22, 23]. 
However, cannabis use does not meet the specificity or strength criteria for causality in that it is neither necessary nor sufficient to cause persistent psychosis in the general population. In summary, frequent cannabis use clearly is associated with subsequent risk for psychotic disorders, particularly schizophrenia. However, though highly suggestive, it is not possible from the available evidence to conclude that this relationship is causal. Rather, based on available evidence, one can only conclude that a relationship exists between heavy, early cannabis use and psychosis - a causal link may occur in either direction. As randomized experiments are not possible, natural experiments in which cannabis use is increased or decreased at a population level by legalization or by prevention programs may help address the issue of causality.

\section{Vulnerability Factors}

There are a number of specific factors that convey increased risk of developing a psychotic disorder after cannabis exposure. A family history of schizophrenia has been shown to increase the risk of psychosis following cannabis exposure $[24,25]$. For example, one study comparing 54 cannabisusing patients with schizophrenia to 71 patients who did not use cannabis found cannabis-using patients to have higher rates of psychosis in their family histories, especially if cannabis use preceded their psychotic symptoms [24]. Another population registry-based study found that family history of schizophrenia increased both the risk of schizophrenia and of acute cannabis-induced psychosis [25]. A history of child abuse (including sexual, physical, and/or emotional abuse) or neglect also increases the risk of developing schizophrenia after cannabis use [26-31]. Two large, population-based, longitudinal studies, in Greece and the Netherlands, both replicated findings that childhood maltreatment and cannabis use precede and are independently associated with increased risk of later psychosis, and that the combination of the 2 risk factors is synergistically associated with an increased risk of psychosis [28]. Finally, being born in and growing up in an urban environment is positively associated with the combination of cannabis use and psychosis. For instance, a large prospective study in and around Munich, Germany, found that the increased risk of psychosis following cannabis use was much greater for those who grew up in the city of Munich than for those raised in surrounding rural areas [32].

One might expect that those vulnerable to developing schizophrenia after cannabis exposure would have greater baseline neurological and cognitive deficits than those less vulnerable. However, the opposite seems to be true. Those who develop schizophrenia following cannabis use tend to have more severe positive psychotic symptoms [24], fewer negative psychotic symptoms [24], fewer cognitive deficits [33], better premorbid functioning [34], acute onset of psychosis without a prodromal period [34], a shorter duration of untreated psychosis [35], and fewer soft signs of neurologic dysfunction $[36,37]$ than those who developed schizophrenia in the absence of cannabis exposure. The findings related to cognitive functioning have been replicated in both first episode and more chronic populations of people with psychotic illness [33]. Explanations for these results have included the idea that disruption of the ECS by exogenous cannabinoids (i.e., THC) during a critical developmental period may interact with specific biological vulnerabilities when psychosis develops following cannabis use, whereas those who develop schizophrenia without cannabis exposure may develop the illness along a different pathway of neurobiological vulnerability.

Regardless of how important cannabis use is in causing the onset of schizophrenia, it is fairly clear that continued cannabis use after diagnosis is associated with more severe positive symptoms and decreased functioning in both first episode and more chronically ill people with schizophrenia [5], and that discontinuing cannabis use improves mood and anxiety symptoms [38], psychotic symptoms [39], and psychosocial functioning [38].

\section{Gene-by-Environment Interactions}

Four genes have emerged as possibly modulating the risk of developing a psychotic illness following exposure to cannabis, in increasing order of replication in the literature: the brain-derived neurotrophic factor $(B D N F)$ gene, the cannabinoid receptor 1 ( $C N R 1)$ gene, the catechol Omethyltransferase (COMT) gene, and AKT1 [40]. A single nucleotide polymorphism (SNP) of the gene encoding brainderived neurotrophic factor, Val66Met (rs6265), has been shown to moderate the relationship between cannabis use and age of onset of psychosis. The interaction may be limited to females for unknown reasons; female cannabis users who carry the Val/Val polymorphism displayed psychotic symptoms on average 7 years earlier than female Met carriers [41]. CNR1 encodes the cannabinoid receptor $1\left(\mathrm{CB}_{1} \mathrm{R}\right)$, which is widespread throughout the central nervous system (CNS), including the brain. In a functional magnetic resonance imaging study of working memory in healthy subjects, those carrying a $\mathrm{G}$ allele in a SNP (rs 1406977) of CNR1 associated with lower receptor expression levels in the prefrontal cortex who also had a history of cannabis use demonstrated decreased working memory and abnormally increased ventromedial prefrontal connectivity than those with AA alleles, as well as to those with a $\mathrm{G}$ allele but no history of cannabis use [42]. A variation of $C N R I$ involving AAT triplet repeats has been associated with a "hebephrenic" type of schizophrenia, characterized by prominent negative symptoms and disorganization $[43,44]$. Catechol O-methyltransferase is one of the enzymes responsible for degrading catecholamines, including dopamine. A Val158Met SNP (rs4680) in COMT has been found to increase the risk of schizophrenia amongst people 
with premorbid cannabis use [45], although this finding has not been consistently replicated [46]. The Val/Val genotype has also been associated with increased symptom severity in individuals with schizophrenia who are using cannabis [45]. Two studies found a 3-way interaction between the COMT SNP, cannabis use, and a history of child abuse. Carriers of the COMT Val/Val genotype who were exposed to both childhood abuse and cannabis had more severe psychotic symptoms, whereas amongst carriers of a Met allele, childhood abuse was associated with more severe psychotic symptoms in noncannabis users $[26,27]$. $A K T 1$ encodes for a protein kinase involved in second messenger transduction at the $\mathrm{CB}_{1} \mathrm{R}$, and has also been implicated in increasing the risk for psychosis in cannabis users. An SNP (rs2494732) has been associated with a greatly increased risk of developing a psychotic disorder amongst cannabis users: compared with $\mathrm{T} / \mathrm{T}$ carriers, $\mathrm{C} / \mathrm{C}$ carriers demonstrated 7-fold odds of developing psychosis [47]. In a study examining potential mechanisms behind this gene-by-cannabis exposure interaction, it was found that subjects with schizophrenia carrying the $\mathrm{C} / \mathrm{C}$ genotype with premorbid cannabis use performed worse on cognitive measures of sustained attention as than $\mathrm{C} / \mathrm{C}$ genotype subjects without premorbid cannabis use, even after longterm abstinence from cannabis. T/T carriers with cannabis use performed as well or better than T/T carriers without cannabis use, suggesting a differential effect on brain functioning and psychosis risk of cannabis use depending on underlying genetic vulnerability [48].

\section{The ECS and Psychosis}

The ECS comprises 2 cannabinoid receptors, $\mathrm{CB}_{1} \mathrm{R}$ in the $\mathrm{CNS}$ and $\mathrm{CB}_{2} \mathrm{R}$ in the periphery [49]; 2 main endogenous ligands, anandamide and arachidonoylglycerol (2-AG) [50]; 2 main endocannabinoid synthesizing enzymes, $N$-acyl phosphatidylethanolamine phospholipase and diacylglycerol lipase; and 2 degradation enzymes, fatty acid amide hydrolase and monoacylglycerol lipase [51]. The system is involved in complex regulation of emotion, reward, and cognition, and has been implicated in both inflammatory and neuroprotective processes [51]. 2-AG is a full agonist at both $\mathrm{CB}_{1} \mathrm{R}$ and $\mathrm{CB}_{2} \mathrm{R}$, whereas anandamide is a partial agonist at $\mathrm{CB}_{1} \mathrm{R}$. Both 2-AG and anandamide are retrograde messengers; they are released postsynaptically and act presynaptically to inhibit release of many excitatory and inhibitory neurotransmitters, including dopamine, glutamate, and $\gamma$-aminobutyric acid (GABA) [52]. Several other molecules also act at cannabinoid receptors, including $\mathrm{N}$ arachidonoyl-dopamine, noladin ether, oleamide, $\mathrm{N}$ oleoylethanolamide, $N$-palmitoylethanolamide, and virodhamine. In addition, endocannabinoids may also modulate neuroplasticity and exert other effects by acting at transient receptor potential vanilloid 1channels, G protein-coupled receptor 55, and peroxisome proliferator-activated receptors $\alpha$ and $\gamma[53] . \mathrm{CB}_{1} \mathrm{R}$ expression is high in brain areas with a greater density of dopaminergic neurons, such as frontal regions, basal ganglia, cerebellum, hippocampus, amygdala, and substantia nigra, and interactions between the endocannabinoid and dopaminergic systems have been thought to play a role in the relationships between cannabinoids and psychosis [54]. $\mathrm{CB}_{1} \mathrm{R}$ are expressed on GABA-ergic and glutamatergic axon terminals in the hippocampus where they modulate long-term potentiation, which is involved in memory and plasticity [55]. The effect of $\mathrm{CB}_{1} \mathrm{R}$ agonists on long-term potentiation may be biphasic, producing enhancement at low concentrations and inhibition at higher concentrations; vanilloid receptors, which are co-localized with $\mathrm{CB}_{1} \mathrm{R}$, may play a role in this modulatory function [56].

Although 2-AG may be more plentiful in the CNS, anandamide has received particular attention in the literature linking the ECS to psychosis [57]. Anandamide was found to be elevated in the cerebrospinal fluid (CSF) of people experiencing prodromal symptoms of schizophrenia compared with healthy controls, but lower anandamide levels correlated with a greater risk of transition to psychosis within the prodromal population [58]. Furthermore, anandamide has been found to be elevated in the CSF of people experiencing both chronic and acute psychotic symptoms relative to healthy controls $[59,60]$. In turn, amongst those with psychosis, CSF anandamide levels are inversely related to the severity of psychotic symptoms [59]. These findings have been interpreted as indicating that anandamide may be part of a natural compensatory mechanism for psychosis in the brain, such that it is elevated in response to onset of psychosis, and the degree of elevation determines the degree of antipsychotic buffering - an interpretation that remains highly speculative. CSF anandamide levels decrease when people with psychosis are treated with typical antipsychotic medications with high dopaminergic blockade, but not when they are treated with newer atypical agents with proportionally greater serotonergic antagonism [59]. People with schizophrenia and a history of chronic cannabis use do not have elevation in CSF anandamide levels [61], suggesting that cannabis use may suppress the brain's natural ability to temper a psychotic process, and pointing towards a potential biological mechanism underlying the cannabis-psychosis link in vulnerable individuals. In healthy volunteers who regularly use cannabis, CSF anandamide levels are decreased and 2-AG levels are increased compared with infrequent cannabis users [62]. In addition, amongst cannabis users, CSF anandamide levels are inversely correlated with persisting psychotic symptoms when cannabis-free, and higher CSF anandamide levels are associated with a lower risk of psychotic symptoms acutely following cannabis use [62].

In summary, these complex and seemingly contradictory findings can be parsed into a compelling and plausible, albeit still preliminary and speculative, biological mechanism for 
how the ECS relates to psychosis. Higher levels of $\mathrm{CB}_{1} \mathrm{R}$, such as via the full agonist endocannabinoid 2-AG or through exogenous cannabinoids such as THC, may directly and indirectly (e.g., via affecting GABAergic transmission) contribute to dopaminergic dysregulation in brain areas important to psychosis, such as frontal cortical areas and the hippocampus [63]. Anandamide may play an important regulatory role in this system, possibly through its partial agonism at the $\mathrm{CB}_{1} \mathrm{R}$ and/or by affecting neuroplasticity. Because anandamide seems to rise in psychosis, which then, in turn, correlates with a reduction in psychotic symptoms, it could be part of a negative feedback system on psychotic processes in the brain. Exogenous cannabinoid exposure may, over time, dysregulate or attenuate this natural antipsychotic compensatory system.

\section{Cannabinoids and Treatment}

THC, the primary psychoactive constituent of cannabis, is a partial agonist at the $\mathrm{CB}_{1} \mathrm{R}$. THC increases extracellular dopamine and glutamate and decreases GABA concentrations in the prefrontal cortex [63]. THC binds more tightly to the receptor and dissociates more slowly than natural endogenous ligands. Psychotomimetic effects of THC are well established, and include paranoia, grandiosity, disorganization, and alterations in sensory perception. When administered to healthy subjects in a laboratory setting, THC produced positive and negative psychotic symptoms, euphoria, anxiety, and impairments in attention, working memory, and verbal recall $[64,65]$. People with schizophrenia exhibit more severe psychotic symptoms in response to THC than healthy controls [66]. Findings have been mixed as to whether antipsychotics attenuate the psychotomimetic effects of THC in healthy subjects $[67,68]$, and chronic antipsychotic treatment does not fully protect individuals with schizophrenia from symptom exacerbation after THC exposure [66]. Forms of cannabis that contain a higher THC content, such as the "skunk-like" or sinsemilla cannabis, commonly used in theUK, have been shown to cause more severe psychotic symptoms and increase the risk for schizophrenia to a greater degree $[69,70]$. More recently, synthetic cannabinoids, which consist of an array of compounds, many of which are full agonists at the $\mathrm{CB}_{1} \mathrm{R}$, have been associated with severe, acute psychosis, and agitation, among a host of other physical and psychological problems [71]. Because of the ability of THC to induce a psychotic syndrome, as well as evidence associating cannabis use with the development of schizophrenia, the potential antipsychotic properties of cannabinoid-based compounds that may counter the effects of THC have engendered great interest. One of the most commonly studied compounds has been rimonabant, a $\mathrm{CB}_{1} \mathrm{R}$ antagonist, initially released in the European Union as an anorectic for the treatment of obesity, but not approved by the Food and Drug Administration and pulled from European Union markets owing to potentially serious adverse effects on mood. Rimonabant has been shown to normalize psychotic-like behaviors in animal models of schizophrenia [72, 73]. In a small, 16-week, placebo-controlled, double-blind, randomized trial of 15 obese patients with schizophrenia, which was terminated when rimonabant was withdrawn from the European market, rimonabant $20 \mathrm{mg}$ per day decreased anxiety, depression and hostility compared with placebo, but did not improve negative symptoms or psychosis [74]. In addition, rimonabant improved a probabilistic test of reward learning, but performance was inferior to placebo on a standard cognitive test battery [75].

Another important constituent of cannabis is cannabidiol (CBD), which has very low affinity for and may be a weak partial agonist at the $\mathrm{CB}_{1} \mathrm{R}$. CBD also inhibits the reuptake and degradation of anandamide, thereby increasing anandamide levels in the CNS [76]. This may have important implications in that anandamide has recently been found to modulate synaptic neuroplasticity via activity at transient receptor potential vanilloid type 1 receptors $[77,78]$. As it has been more widely studied, and because of its greater therapeutic promise, we focus more attention on CBD.

\section{CBD}

Whereas CBD is very well tolerated and has few psychoactive effects on its own [79], it counteracts several effects of THC and other $\mathrm{CB}_{1} \mathrm{R}$ agonists in healthy subjects [80], including anxiety, euphoria, and psychosis [3, 73, 81]. CBD has also been shown to attenuate THC-induced memory impairment [80]. However, in mice and rats, CBD inhibits metabolism of THC, so that pretreatment with CBD can increase brain concentrations of THC and thereby potentiate its behavioral effects [82]. In a series of functional magnetic resonance imaging experiments in which $\mathrm{CBD}$ and $\mathrm{THC}$ were administered to healthy human subjects, the 2 compounds produced largely opposite patterns of activation during tasks involving memory, emotional processing, salience, and response inhibition in key brain regions implicated in schizophrenia, such as the striatum, prefrontal cortex, and hippocampus [3, 81]. There is also evidence that using cannabis with a higher $\mathrm{CBD} /$ THC ratio is associated with a lower risk of psychotic disorders, subthreshold psychotic symptoms, and cognitive changes related to psychosis [3], from both studies analyzing THC and CBD concentrations in hair samples [83, 84], and from a study analyzing the content of the cannabis that subjects reported regularly using [85]. Cannabis users have been found to have evidence for neurotoxicity in the prefrontal cortex based on lower levels of $N$-acetylaspartic acid measured by proton magnetic spectroscopy, which reflects neuronal integrity [86]. A higher ratio of CBD to THC concentrations in hair samples of cannabis users was associated with higher levels of $\mathrm{N}$-acetylaspartic acid in the putamen/globus pallidus region [86], as well as larger hippocampal volume [87, 88]. 
The potential antipsychotic properties of CBD independent of cannabis use have garnered increasing attention recently. Preclinical animal studies have shown that CBD normalizes behaviors in rodent models for schizophrenia [72, 89], such as social interaction and performance on novel object recognition tests. In one study, psychotic-like behaviors induced in mice through $N$-methyl-D-aspartate receptor antagonism with the phencyclidine-like drug, dizocilpine (MK-801), were normalized by either clozapine or CBD administration [90]. CBD also normalized markers of glial cell dysfunction and inflammation induced by $N$-methyl-D-aspartate receptor antagonist administration, suggesting that CBD may have antiinflammatory and neuroprotective properties [90].

Five published clinical studies have examined the antipsychotic effects of CBD in humans. In 1995, Zuardi et al. [91] published a case report in which a patient with treatmentresistant schizophrenia was administered up to $1500 \mathrm{mg}$ orally per day of CBD for 26 days, with symptomatic improvement and no adverse effects. In a follow-up report, the same group studied the effects of up to $1280 \mathrm{mg}$ orally per day of CBD for 4 weeks in 3 patients with treatment-resistant psychosis. Only 1 of the 3 patients improved, but none had adverse effects [92]. In another study, CBD up to $600 \mathrm{mg}$ orally per day for 6 weeks significantly reduced psychotic symptoms in 6 patients with Parkinson's disease and was well tolerated [93]. Hallak et al. studied the acute effects of CBD on selective attention, as well as electrodermal responsiveness to auditory stimuli, in 28 medication-free schizophrenic patients. They found no differences on any measure between patients who received a single oral dose of CBD $300 \mathrm{mg}, \mathrm{CBD} 600 \mathrm{mg}$, or placebo [94]. Finally, the largest clinical study of the effects of CBD on psychosis to date was a 4-week, double-blind, randomized trial comparing CBD, up to $800 \mathrm{mg}$ per day $(n=20)$, to the antipsychotic amisulpride $(n=19)$. Both groups significantly improved on ratings of positive and negative symptoms with no significant differences between treatment groups. However, the CBD group experienced significantly fewer side effects, including prolactin elevations, extrapyramidal symptoms, and weight gain [76]. Further analysis showed that CBD treatment led to a significant increase in serum anandamide levels, which correlated with improvement in symptoms [76]. If replicated in larger samples, such findings could suggest that CBD may have significant antipsychotic properties with superior tolerability to currently available antipsychotic drugs [73]. However, further larger scale, placebo-controlled trials are needed to determine whether CBD would make an effective and safe alternative to available antipsychotic treatments.

\section{Future Directions}

The evidence for an association between cannabis use and schizophrenia is compelling, as is the evidence that THC and synthetic cannabinoids can provoke symptoms of schizophrenia in healthy subjects and exacerbate symptoms in patients with schizophrenia. THC has multiple actions that could disrupt brain development in vulnerable adolescents, including impairment of neuroplasticity, dysregulation of dopamine and glutamate signaling, and, possibly, neurotoxicity. Although still highly speculative, it is an intriguing hypothesis that cannabis use in adolescence could contribute to the onset of a distinct form of schizophrenia which differs from the cannabis-independent neurodevelopmental pathway to schizophrenia characterized by more gradual onset, greater cognitive impairment, and functional disability. CBD attenuates many of the adverse effects of THC, including psychosis and some elements of cognitive impairment. CBD may also be neuroprotective, based on observations of individuals using cannabis with high or low relative concentrations of CBD. Whereas simultaneous administration of CBD and THC appears to protect against THC toxicity in humans, rodent studies have found that pretreatment with CBD can potentiate THC effects by inhibiting its metabolism. This potential complication therefore requires study in humans, as it could have important implications for administration of CBD in cannabis users. Many potential clinical and preventive strategies follow from the accumulated literature. Increasing decriminalization and legalization of cannabis may present opportunities to investigate ways to reduce the harm of cannabis use through regulation. For example, government regulators might monitor the ratio of $\mathrm{CBD}$ to $\mathrm{THC}$ in cannabis products, allowing researchers to determine whether requiring an increased ratio of CBD to THC might reduce the risk of harm in vulnerable individuals. It is also possible that biomarkers, such as CSF anandamide or genetic profiling based on AKT1, COMT, or CNR1 might identify individuals at high risk for or early in the course of psychosis who might benefit from CBD or other endocannabinoid-targeted interventions and may even not require chronic antipsychotic treatment. Whether CBD might represent a general alternative or augmentative therapeutic strategy for patients with schizophrenia will require much additional research.

Required Author Forms Disclosure forms provided by the authors are available with the online version of this article.

\section{References}

1. Kendler KS, Gallagher TJ, Abelson JM, Kessler RC. Lifetime prevalence, demographic risk factors, and diagnostic validity of nonaffective psychosis as assessed in a US community sample. The National Comorbidity Survey. Arch Gen Psychiatry 1996;52: 1022-1031.

2. Tandon R, Gaebel W, Barch DM, et al. Definition and description of schizophrenia in the DSM-5. Schizophr Res 2013;150:3-10. 
3. Iseger TA, Bossong MG. A systematic review of the antipsychotic properties of cannabidiol in humans. Schizophr Res 2015;162:153161.

4. Radhakrishnan R, Wilkinson ST, D'Souza DC. Gone to pot-a review of the association between cannabis and psychosis. Front Psychiatry 2014;5:54.

5. Clausen L, Hjorthoj CR, Thorup A, et al. Change in cannabis use, clinical symptoms and social functioning among patients with firstepisode psychosis: a 5-year follow-up study of patients in the OPUS trial. Psychol Med 2014;44:117-126.

6. Andreasson S, Allebeck P, Engstrom A, Rydberg U. Cannibis and schizophrenia: A longitudinal study of Swedish conscripts. Lancet 1987;2:1483-1485.

7. Moore THM, Zammit S, Lingford-Hughes A, et al. Cannabis use and risk of psychotic or affective mental health outcomes: a systematic review. Lancet 2007;370:319-328.

8. Minozzi S, Davoli M, Bargagli AM, Amato L, Vecchi S, Perucci CA. An overview of systematic reviews on cannabis and psychosis: discussing apparently conflicting results. Drug Alcohol Rev 2010;29:304-317.

9. Rowe AR, Mercer L, Casetti V, et al. Dementia praecox redux: A systematic review of the nicotinic receptor as a target for cognitive symptoms of schizophrenia. J Psychopharmacol 2015;29:197-211.

10. Power RA, Verweij KJ, Zuhair M, et al. Genetic predisposition to schizophrenia associated with increased use of cannabis. Mol Psychiatry 2014;19:1201-1204.

11. Large M, Sharma S, Compton MT, Slade T, Nielssen O. Cannabis use and earlier onset of psychosis: a systematic meta-analysis. Arch Gen Psychiatry 2011;68:555-561.

12. Schimmelmann BG, Conus P, Cotton SM, et al. Cannabis use disorder and age at onset of psychosis-a study in first-episode patients. Schizophr Res 2011;129:52-56.

13. Stefanis NC, Dragovic M, Power BD, Jablensky A, Castle D, Morgan VA. Age at initiation of cannabis use predicts age at onset of psychosis: the 7- to 8-year trend. Schizophr Bull 2013;39:251254.

14. van Gastel WA, Wigman JT, Monshouwer K, et al. Cannabis use and subclinical positive psychotic experiences in early adolescence: findings from a Dutch survey. Addiction 2012;107:381-387.

15. Ferdinand RF, Sondeijker F, van der Ende J, Selten JP, Huizink A, Verhulst FC. Cannabis use predicts future psychotic symptoms, and vice versa. Addiction 2005;100:612-618.

16. Henquet C, Krabbendam L, Spauwen J, et al. Prospective cohort study of cannabis use, predisposition for psychosis, and psychotic symptoms in young people. BMJ 2005;330:1-5.

17. McGrath J, Welham J, Scott J, et al. Association between cannabis use and psychosis-related outcomes using sibling pair analysis in a cohort of young adults. Arch Gen Psychiatry 2010;67:440-447.

18. Macleod J, Oakes R, Copello A, et al. Psychological and social sequelae of cannabis and other illicit drug use by young people: a systematic review of longitudinal, general population studies. Lancet 2004;363:1579-1588.

19. Gage SH, Zammit S, Hickman M. Stronger evidence is needed before accepting that cannabis plays an important role in the aetiology of schizophrenia in the population. F1000 Med Rep. 2013;5:2

20. Fergusson DM, Horwood LJ, Swain-Campbell NR. Cannabis dependence and psychotic symptoms in young people. Psychol Med 2003;33:15-21.

21. Manrique-Garcia E, Zammit S, Dalman C, Hemmingsson T, Andreasson S, Allebeck P. Cannabis, schizophrenia and other non-affective psychoses: 35 years of follow-up of a populationbased cohort. Psychol Med 2012;42:1321-1328.

22. Hall W, Degenhardt L. Cannabis use and the risk of developing a psychotic disorder. World Psychiatry 2008;7:68-71.
23. Degenhardt L, Hall W. Is cannabis a contributory cause of psychosis? Can J Psychiatry2006;51:556-565.

24. Bersani G, Orlandi V, Kotzalidis GD, Pancheri P. Cannabis and schizophrenia: impact on onset, course, psychopathology and outcomes. Eur Arch Psychiatry Clin Neurosci 2002;252:86-92.

25. Arendt M, Mortensen PB, Rosenberg R, Pedersen CB, Waltoft BL. Familial predisposition for psychiatric disorder: comparison of subjects treated for cannabis-induced psychosis and schizophrenia. Arch Gen Psychiatry 2008;65:1269-1274.

26. Alemany S, Arias B, Fatjo-Vilas M, et al. Psychosis-inducing effects of cannabis are related to both childhood abuse and COMT genotypes. Acta Psychiatr Scand 2014;129:54-62.

27. Vinkers CH, Van Gastel WA, Schubart CD, et al. The effect of childhood maltreatment and cannabis use on adult psychotic symptoms is modified by the COMT Val(1)(5)(8)Met polymorphism. Schizophr Res 2013;150:303-311.

28. Konings M, Stefanis N, Kuepper R, et al. Replication in two independent population-based samples that childhood maltreatment and cannabis use synergistically impact on psychosis risk. Psychol Med 2012:42:149-159.

29. Houston JE, Murphy J, Shevlin M, Adamson G. Cannabis use and psychosis: re-visiting the role of childhood trauma. Psychol Med 2011:41:2339-2348.

30. Harley M, Kelleher I, Clarke M, et al. Cannabis use and childhood trauma interact additively to increase the risk of psychotic symptoms in adolescence. Psychol Med 2010;40:1627-1634.

31. Compton MT, Furman AC, Kaslow NJ. Preliminary evidence of an association between childhood abuse and cannabis dependence among African American first-episode schizophrenia-spectrum disorder patients. Drug Alcohol Depend 2004;76:311-316.

32. Kuepper R, van Os J, Lieb R, Wittchen HU, Henquet C. Do cannabis and urbanicity co-participate in causing psychosis? Evidence from a 10-year follow-up cohort study. Psychol Med 2011;41:21212129.

33. Yucel M, Bora E, Lubman DI, et al. The impact of cannabis use on cognitive functioning in patients with schizophrenia: a metaanalysis of existing findings and new data in a first-episode sample. Schizophr Bull 2012;38:316-330.

34. Compton MT, Broussard B, Ramsay CE, Stewart T. Pre-illness cannabis use and the early course of nonaffective psychotic disorders: associations with premorbid functioning, the prodrome, and mode of onset of psychosis. Schizophr Res 2011;126:71-76.

35. Burns JK. Cannabis use and duration of untreated psychosis: a systematic review and meta-analysis. Curr Pharm Des 2012;18: 5093-5104.

36. Loberg EM, Helle S, Nygard M, Berle JO, Kroken RA, Johnsen E. The cannabis pathway to non-affective psychosis may reflect less neurobiological vulnerability. Front Psychiatry 2014;5:159.

37. Ruiz-Veguilla M, Callado LF, Ferrin M. Neurological soft signs in patients with psychosis and cannabis abuse: a systematic review and meta-analysis of paradox. Curr Pharm Des 2012;18:51565164.

38. Barrowclough C, Gregg L, Lobban F, Bucci S, Emsley R. The impact of cannabis use on clinical outcomes in recent onset psychosis. Schizophr Bull 2015;41:382-390.

39. Stone JM, Fisher HL, Major B, et al. Cannabis use and first-episode psychosis: relationship with manic and psychotic symptoms, and with age at presentation. Psychol Med 2014;44:499-506.

40. Decoster J, van Os J, Myin-Germeys I, De Hert M, van Winkel R. Genetic variation underlying psychosis-inducing effects of cannabis: critical review and future directions. Curr Pharm Des 2012;18: 5015-5023.

41. Decoster J, van Os J, Kenis G, et al. Age at onset of psychotic disorder: cannabis, BDNF Val66Met, and sex-specific models of gene-environment interaction. Am J Med Genet B Neuropsychiatr Genet 2011;156B:363-369. 
42. Colizzi M, Fazio L, Ferranti L, et al. Functional genetic variation of the cannabinoid receptor 1 and cannabis use interact on prefrontal connectivity and related working memory behavior. Neuropsychopharmacology 2015;40:640-649.

43. Chavarria-Siles I, Contreras-Rojas J, Hare E, et al. Cannabinoid receptor 1 gene (CNR1) and susceptibility to a quantitative phenotype for hebephrenic schizophrenia. Am J Med Genet B Neuropsychiatr Genet 2008;147:279-284.

44. Ujike H, Takaki M, Nakata K, et al. CNRI, central cannabinoid receptor gene, associated with susceptibility to hebephrenic schizophrenia. Mol Psychiatry 2002;7:515-518.

45. Ermis A, Erkiran M, Dasdemir S, et al. The relationship between catechol-O-methyltransferase gene Val158Met (COMT) polymorphism and premorbid cannabis use in Turkish male patients with schizophrenia. In Vivo 2015;29:129-132.

46. Zammit S, Owen MJ, Evans J, Heron J, Lewis G. Cannabis, COMT and psychotic experiences. Br J Psychiatry 2011;199:380-385.

47. Di Forti M, Iyegbe C, Sallis H, et al. Confirmation that the AKT1 (rs2494732) genotype influences the risk of psychosis in cannabis users. Biol Psychiatry 2012;72:811-816.

48. van Winkel R, van Beveren NJ, Simons C, Genetic R, Outcome of Psychosis I. AKT1 moderation of cannabis-induced cognitive alterations in psychotic disorder. Neuropsychopharmacology 2011;36: 2529-2537.

49. Muller-Vahl KR, Emrich HM. Cannabis and schizophrenia: towards a cannabinoid hypothesis of schizophrenia. Expert Rev Neurother 2008;8:1037-1048.

50. Kucerova J, Tabiova K, Drago F, Micale V. Therapeutic potential of cannabinoids in schizophrenia. Recent Pat CNS Drug Discov 2014;9:13-25.

51. Bioque M, Garcia-Bueno B, Macdowell KS, et al. Peripheral endocannabinoid system dysregulation in first-episode psychosis. Neuropsychopharmacology 2013;38:2568-2577.

52. Wenzel JM, Cheer JF. Endocannabinoid-dependent modulation of phasic dopamine signaling encodes external and internal rewardpredictive cues. Front Psychiatry 2014;5:118.

53. Pertwee RG. Receptors and channels targeted by synthetic cannabinoid receptor agonists and antagonists. Curr Med Chem 2010;17: 1360-1381.

54. van Winkel R, Kuepper R. Epidemiological, neurobiological, and genetic clues to the mechanisms linking cannabis use to risk for nonaffective psychosis. Annu Rev Clin Psychol 2014;10:767-791.

55. Monory K, Polack M, Remus A, Lutz B, Korte M. Cannabinoid $\mathrm{CB} 1$ receptor calibrates excitatory synaptic balance in the mouse hippocampus. J Neurosci 2015;35:3842-3850.

56. Tahmasebi L, Komaki A, Karamian R, et al. The interactive role of cannabinoid and vanilloid systems in hippocampal synaptic plasticity in rats. Eur J Pharmacol 2015;757:68-73.

57. Leweke FM. Anandamide dysfunction in prodromal and established psychosis. Curr Pharm Des 2012;18:5188-5193.

58. Koethe D, Giuffrida A, Schreiber D, et al. Anandamide elevation in cerebrospinal fluid in initial prodromal states of psychosis. Br J Psychiatry 2009;194:371-372.

59. Giuffrida A, Leweke FM, Gerth CW, et al. Cerebrospinal anandamide levels are elevated in acute schizophrenia and are inversely correlated with psychotic symptoms. Neuropsychopharmacology 2004;29:2108-2114

60. Leweke FM, Giuffrida A, Wurster U, Emrich HM, Piomelli D. Elevated endogenous cannabinoids in schizophrenia. Neuroreport 1999;10:1665-1669.

61. Leweke FM, Giuffrida A, Koethe D, et al. Anandamide levels in cerebrospinal fluid of first-episode schizophrenic patients: impact of cannabis use. Schizophr Res 2007;94:29-36.

62. Morgan CJ, Page E, Schaefer C, et al. Cerebrospinal fluid anandamide levels, cannabis use and psychotic-like symptoms. Br J Psychiatry 2013;202:381-382.
63. Pistis M, Ferraro L, Pira L, et al. Delta(9)-tetrahydrocannabinol decreases extracellular GABA and increases extracellular glutamate and dopamine levels in the rat prefrontal cortex: an in vivo microdialysis study. Brain Res 2002;948:155-158.

64. D'Souza DC, Perry E, MacDougall L, et al. The psychotomimetic effects of intravenous delta-9-tetrahydrocannabinol in healthy individuals: implications for psychosis. Neuropsychopharmacology 2004;29:1558-1572.

65. Morrison PD, Zois V, McKeown DA, et al. The acute effects of synthetic intravenous Delta9-tetrahydrocannabinol on psychosis, mood and cognitive functioning. Psychol Med 2009;39:1607-1616.

66. D'Souza DC, Abi-Saab WM, Madonick S, et al. Delta-9tetrahydrocannabinol effects in schizophrenia: implications for cognition, psychosis, and addiction. Biol Psychiatry 2005;57:594608.

67. Liem-Moolenaar M, te Beek ET, de Kam ML, et al. Central nervous system effects of haloperidol on THC in healthy male volunteers. J Psychopharmacol 2010;24:1697-1708.

68. D'Souza DC, Braley G, Blaise R, et al. Effects of haloperidol on the behavioral, subjective, cognitive, motor, and neuroendocrine effects of Delta-9-tetrahydrocannabinol in humans. Psychopharmacology 2008;198:587-603.

69. Di Forti M, Marconi A, Carra E, et al. Proportion of patients in south London with first-episode psychosis attributable to use of high potency cannabis: a case-control study. Lancet Psychiatry 2015;2:233-238.

70. Di Forti M, Sallis H, Allegri F, et al. Daily use, especially of highpotency cannabis, drives the earlier onset of psychosis in cannabis users. Schizophr Bull 2014;40:1509-1517.

71. van Amsterdam J, Brunt T, van den Brink W. The adverse health effects of synthetic cannabinoids with emphasis on psychosis-like effects. J Psychopharmacol 2015;29:254-263.

72. Levin R, Almeida V, Peres FF, et al. Antipsychotic profile of cannabidiol and rimonabant in an animal model of emotional context processing in schizophrenia. Curr Pharm Des 2012;18:49604965.

73. Roser P, Haussleiter IS. Antipsychotic-like effects of cannabidiol and rimonabant: systematic review of animal and human studies. Curr Pharm Des 2012;18:5141-5155.

74. Kelly DL, Gorelick DA, Conley RR, et al. Effects of the cannabinoid-1 receptor antagonist rimonabant on psychiatric symptoms in overweight people with schizophrenia: a randomized, double-blind, pilot study. J Clin Psychopharmacol 2011;31:86-91.

75. Boggs DL, Kelly DL, McMahon RP, et al. Rimonabant for neurocognition in schizophrenia: a 16-week double blind randomized placebo controlled trial. Schizophr Res 2012;134:207-210.

76. Leweke FM, Piomelli D, Pahlisch F, et al. Cannabidiol enhances anandamide signaling and alleviates psychotic symptoms of schizophrenia. Transl Psychiatry 2012;2:e94.

77. Yang K, Lei G, Xie YF, MacDonald JF, Jackson MF. Differential regulation of NMDAR and NMDAR-mediated metaplasticity by anandamide and 2-AG in the hippocampus. Hippocampus 2014;:24:1601-1614.

78. Chavez AE, Chiu CQ, Castillo PE. TRPV1 activation by endogenous anandamide triggers postsynaptic long-term depression in dentate gyrus. Nat Neurosci 2010;13:1511-1518.

79. Martin-Santos R, Crippa JA, Batalla A, et al. Acute effects of a single, oral dose of d9-tetrahydrocannabinol (THC) and cannabidiol (CBD) administration in healthy volunteers. Curr Pharm Des 2012;18:4966-4979.

80. Englund A, Morrison PD, Nottage J, et al. Cannabidiol inhibits THC-elicited paranoid symptoms and hippocampal-dependent memory impairment. J Psychopharmacol 2013;27:19-27.

81. Bhattacharyya S, Morrison PD, Fusar-Poli P, et al. Opposite effects of delta-9-tetrahydrocannabinol and cannabidiol on human brain 
function and psychopathology. Neuropsychopharmacology 2010;35:764-774.

82. Klein C, Karanges E, Spiro A, et al. Cannabidiol potentiates Delta(9)-tetrahydrocannabinol (THC) behavioural effects and alters THC pharmacokinetics during acute and chronic treatment in adolescent rats. Psychopharmacology 2011;218:443-457.

83. Morgan CJ, Gardener C, Schafer G, et al. Sub-chronic impact of cannabinoids in street cannabis on cognition, psychotic-like symptoms and psychological well-being. Psychol Med 2012;42:391400.

84. Morgan CJ, Curran HV. Effects of cannabidiol on schizophrenialike symptoms in people who use cannabis. Br J Psychiatry 2008;192:306-307.

85. Schubart CD, Sommer IE, van Gastel WA, Goetgebuer RL, Kahn RS, Boks MP. Cannabis with high cannabidiol content is associated with fewer psychotic experiences. Schizophr Res 2011;130:216221.

86. Hermann D, Sartorius A, Welzel H, et al. Dorsolateral prefrontal cortex N-acetylaspartate/total creatine (NAA/tCr) loss in male recreational cannabis users. Biol Psychiatry 2007;61:1281-1289.

87. Hermann D, Schneider M. Potential protective effects of cannabidiol on neuroanatomical alterations in cannabis users and psychosis: a critical review. Curr Pharm Des 2012;18:4897-4905.
88. Demirakca T, Sartorius A, Ende G, et al. Diminished gray matter in the hippocampus of cannabis users: possible protective effects of cannabidiol. Drug Alcohol Depend 2011;114:242-245.

89. Schubart CD, Sommer IE, Fusar-Poli P, de Witte L, Kahn RS, Boks MP. Cannabidiol as a potential treatment for psychosis. Eur Neuropsychopharmacol 2014;24:51-64.

90. Gomes FV, Llorente R, Del Bel EA, Viveros MP, Lopez-Gallardo $\mathrm{M}$, Guimaraes FS. Decreased glial reactivity could be involved in the antipsychotic-like effect of cannabidiol. Schizophr Res 2015;164:155-163.

91. Zuardi AW, Morais SL, Guimarães FS, Mechoulam R. Antipsychotic effect of cannabidiol. J Clin Psychiatry 1995;56: 485-486.

92. Zuardi AW, Hallak JE, Dursun SM, et al. Cannabidiol monotherapy for treatment-resistant schizophrenia. J Psychopharmacol 2006;20: 683-686.

93. Zuardi AW, Crippa JAS, Hallak JEC, et al. Cannabidiol for the treatment of psychosis in Parkinson's disease. J Psychopharmacol 2009;23:979-983.

94. Hallak JEC, Machado-de-Sousa JP, Crippa JAS, et al. Performance of schizophrenic patients in the Stroop Color Word Test and electrodermal responsiveness after acute administration of cannabidiol (CBD). Rev Bras Psiquiatr 2010;31:56-61. 